\title{
Characteristic Modes of Slotted Planar Antennas
}

\author{
Nora Mohamed Mohamed-Hicho ${ }^{(1)}$, Eva Antonino-Daviu ${ }^{(1)}$, Marta Cabedo-Fabrés ${ }^{(1)}$, Juan Pablo Ciafardini ${ }^{(2)}$, J. \\ Alberto Bava ${ }^{(2)}$, MiguelFerrando-Bataller ${ }^{(1)}$. \\ ${ }^{(1)}$ Instituto de Telecomunicaciones y Aplicaciones Multimedia, Universitat Politècnica de València, \\ Valencia (46022), Spain, nomomo@upvnet.upv.es \\ (2) Dpto. de Electrotecnia. Facultad de Ingeniería, Universidad Nacional de La Plata. Calle 48 y 116 - La Plata (1900), Argentina.
}

\begin{abstract}
In this paper, a modal analysis of a slot inserted in a finite ground plane is presented, giving physical understanding of its radiation behavior and the interaction effects between the slot and the plane. The Theory of Characteristic Modes (TCM) reveals that for the case of slotted planar antennas the modes of the slot do not appear as independent modes. This paper also shows that the slot resonance fixes the upper frequency of the excited modes in the combined structure formed by the slot and the finite plate. Moreover, the modal analysis demonstrates that the radiation pattern stability and the radiation bandwidth of the slot antenna are directly related with the size of the finite ground plane. The conclusions reached with the application of TCM can be employed to optimize the geometry and the size of the finite plate in order to prevent the excitation of non-desired modes.
\end{abstract}

Index Terms - Characteristic Modes, Magnetic modes, Excitation of Characteristic Modes, Slot Antenna, Antenna Design.

\section{INTRODUCTION}

The utility and consequent success of the Theory of Characteristic Modes (TCM) [1][2] for antenna design, is founded on the physical insight it provides into the phenomena that governs the radiation, especially when used in metallic structures with simple geometries in which currents present predictable schemes. The TCM has demonstrated to be highly useful for the systematic analysis and design of arbitrarily-shaped antennas [3], such as small antennas [4], planar patches [5], monopoles [3] and handset antennas [6], among others, because it reveals information that is otherwise lost by standard electromagnetic modes and their associated simulation techniques. In the last years, Characteristic Modes (CM) have attracted a lot of interest, leading to an exponential increase of the number of publications concerning the use of this modal theory for the analysis and design of antennas.

Modal analysis can be easily performed for the case of electrically small and medium size metallic structures with narrowband characteristics, since only few modes are needed for a full characterization of the radiating behavior of the antenna. However, for complex antenna geometries and antennas with more than one resonator, the number of modes increases (as result of different kinds of coupling) and characteristic currents present quite an unpredictable behavior, so the physical interpretation becomes not so evident. An example of this kind of geometries that combine different resonators are slotted planar antennas.

Moreover, small antennas have received considerable attention in the last decades due the rising requirement on miniaturized products for modern wireless communications. Slot antennas are appealing candidates for that application, due to their low profile, large bandwidth, good radiation efficiency, easy impedance matching, simple structure, and stable performance in the proximity of metallic components [7][8]. In planar antenna design, the inclusion of slots is a common technique for producing band-notch effects [9], achieving size miniaturization [8], generating dual-band and multi-band characteristics [7] and producing circular polarization [10]. In order to achieve the desired effect when the slot is etched in a planar structure, it is important to understand the mechanisms that control radiation in slotted planar antennas.

Commonly, the design of slotted planar antennas becomes a systematic task that lacks of physical insight, since it is carried out by tuning the feed locations and the patch profile through parameter sweeps, or by using automatic optimization techniques. In this paper, it is demonstrated that the Theory of Characteristic Modes can be very profitable for slotted planar antennas design, giving physical understanding of the interaction between the slot and the finite ground plane.

The analysis of slotted planar structures with $\mathrm{CM}$ was first performed by R.F. Harrington in [11] using equivalent magnetic currents. The magnetic characteristic currents were also obtained in [12] for a rectangular aperture in an infinite perfectly conducting plane. After these works, little research has been conducted about the computation of $\mathrm{CM}$ in apertures and slots.

The structure of the paper is the following: Section II presents a magnetic modal analysis of a slot embedded in an infinite ground plane. Section III shows the electric modal analysis of a slot etched in a finite ground plane. Firstly, an isolated finite electrical ground plate is analyzed in order to understand the interaction between the slot and the plane. The modal analysis of section II and III has been carried out by using the Characteristic Modes request of FEKO. Section IV introduces the analysis of the slot antenna when a voltage gap is placed in the middle of the slot. Results of section IV have been obtained using the commercial electromagnetic software CST Microwave Studio. In addition, design 
guidelines for slotted antennas are inferred from the modal analysis performed in this section. The paper finishes with the conclusions.

\section{Modal ANALysis of A SLOt in AN Infinite Plane}

The analysis of slotted planar structures with CM using equivalent magnetic currents was first performed by R.F. Harrington in [11], as previously commented. In this work, it was proved that $\mathrm{CM}$ could be defined for aperture problems considering an admittance operator and the following weighted eigenvalue equation:

$$
B\left(M_{n}\right)=b_{n} G\left(M_{n}\right)
$$

where $b_{n}$ are the real magnetic eigenvalues, $M_{n}$ are the characteristic magnetic currents and $G$ and $B$ are the real and imaginary parts of the admittance operator $Y$ :

$$
Y=G+j B
$$

When a planar structure composed of a resonant slot in an infinite perfectly conducting plane is analyzed from a modal point of view, the magnetic characteristic currents $\left(M_{n}\right)$ for the slot are obtained. It can be proved by applying Babinet's principle [13] that the electric characteristic currents $\left(J_{n}\right)$ of a metallic plate with the same shape and size as the slot are completely dual to these magnetic characteristic currents $\left(M_{n}\right)$ of the slot.

The geometry of the first structure under study is presented in Fig. 1. As seen, the structure is composed by a slot etched in an infinite ground plane. Theoretically, the resonances of the first, second and third magnetic characteristic modes of the slot occurs when its length $L_{S}$ is approximately $\lambda_{S} / 2(4.4 \mathrm{GHz}), \lambda_{S}(8.8 \mathrm{GHz})$ and $3 \lambda_{S} / 2(13.2$ $\mathrm{GHz}$ ), respectively. Fig. 2 depicts the real magnetic eigenvalue versus frequency for the first four modes of the slot. A magnetic mode is at resonance when its associated real magnetic eigenvalue is equal to zero $\left(b_{n}=0\right)$. The magnetic resonant modes have inductive behavior at low frequencies when the real magnetic eigenvalue is less than zero $\left(b_{n}<0\right)$, and a capacitive behavior after the resonance $\left(b_{n}>0\right)$. As observed, the resonance frequencies numerically obtained for the magnetic modes are very close to the theoretical ones. In Fig. 2, the corresponding simulated 3D radiation patterns at resonance for the first three magnetic modes of the slot, are also presented. Fig. 3 shows the magnetic current distribution in the slot for the first three modes displayed in Fig. 2, at their resonance frequency. Results depicted in Fig. 3, evidence the duality that exists between the well-known electric modes of a dipole and the magnetic modes of a slot, since both structures present the same current distribution behavior and identical resonance frequency.

Nevertheless, this duality of the dipole and the slot revealed by the magnetic modal approach, is only valid when an infinite ground plane is considered. In practice slots are etched in finite conducting planes, so the interaction between the slot modes and the finite metallic plane modes should be taken into account, using an electric modal approach. One of the conclusions reached with the modal analysis presented in [14] is that the size of the finite ground plane plays a crucial role in the radiation pattern of slot antennas and in its stability with frequency. Moreover, the radiating bandwidth is also related to the size of the plate, as it will be demonstrated in following sections.

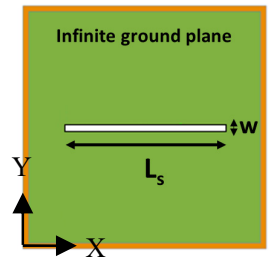

Fig. 1. Geometry and dimensions of the rectangular slot in an infinite ground plane: $L_{S}=33 \mathrm{~mm}$ and $W=1 \mathrm{~mm}$.

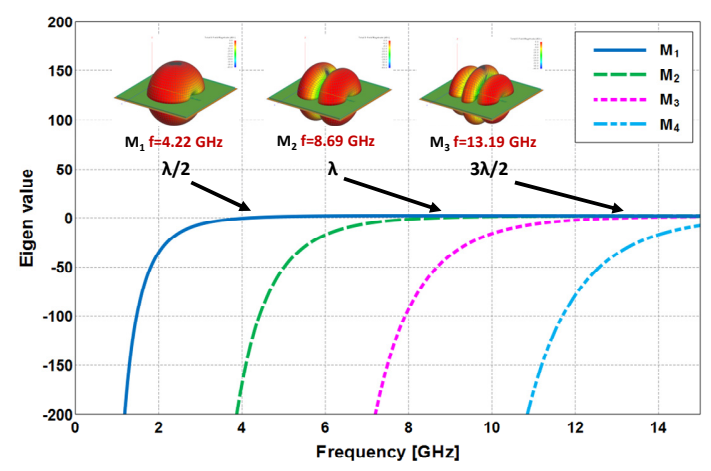

Fig. 2. Eigenvalues versus frequency for the first four modes of a rectangular slot with $L_{S}=33 \mathrm{~mm}$ and $W=1 \mathrm{~mm}$.

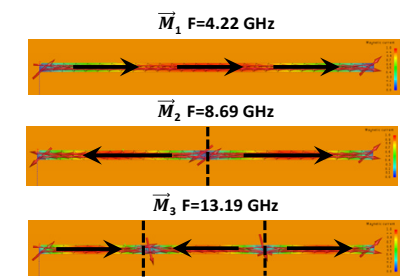

Fig. 3. Distribution of the magnetic characteristic current for the first three modes of a rectangular slot with $L_{S}=33 \mathrm{~mm}$ and $W=1 \mathrm{~mm}$, at their resonant frequency.

\section{Modal ANalysis of a Slotted Planar STRUCTURE}

This section presents a complete analysis of the modal electric currents of a planar structure composed of a slot etched on a finite square plane. The interaction between the corresponding modes of the slot and the ground plane is fully discussed. Fig. 4 shows de geometry and dimensions of the square metallic plate $(L=80 \mathrm{~mm})$ and the slot $\left(L_{S}=33 \mathrm{~mm}\right.$, $W=1 \mathrm{~mm}$ ) under consideration.

Firstly, an isolated ground plate is going to be studied in order to understand later the CM results obtained with the combined structure composed by the slot and the square plate. Fig. 5 shows the characteristic angle versus frequency for the first eleven modes of the square metallic plate. Two kinds of characteristic modes can be identified in this figure, 
resonant and non-resonant electric modes. Resonant modes have capacitive behavior at low frequencies, resonate when its associated characteristic angle is equal to $180^{\circ}$, and become inductive beyond their resonance. Non-resonant modes or inductive modes (like mode $J_{0}$ ) do not resonate. Due to the symmetry of the metallic plate, there are degenerate pairs of modes, such as pairs $J_{1-} J_{l}$ ' and $J_{4}-J_{4}$ ', that resonate at exactly the same frequency. Theoretically, the resonance of the first two electric degenerated modes of the square metallic plane $\left(J_{l}-J_{1}{ }^{\prime}\right)$ occurs when the length $L$ is approximately $\lambda / 2(\mathrm{f}=1.85 \mathrm{GHz})$.

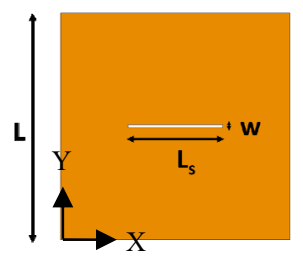

Fig. 4. Geometry and dimensions of the square metallic plate with the slot: $L_{S}=33 \mathrm{~mm}, W=1 \mathrm{~mm}$, and $L=80 \mathrm{~mm}$.

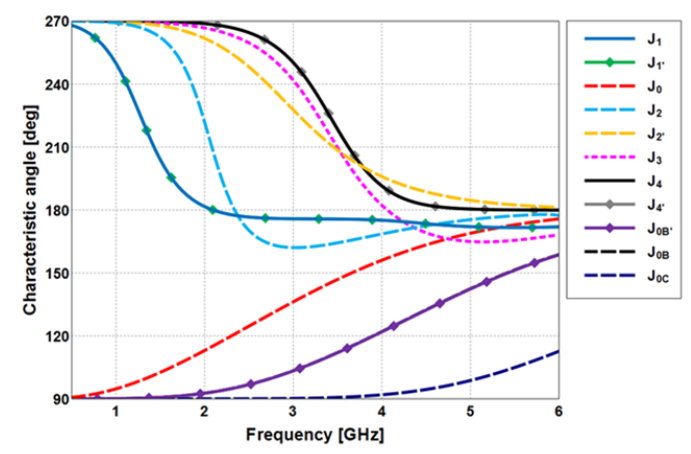

Fig. 5. Characteristic angle versus frequency for the first eleven electric characteristic modes of the square metallic plate of length $L=80 \mathrm{~mm}$ without slot

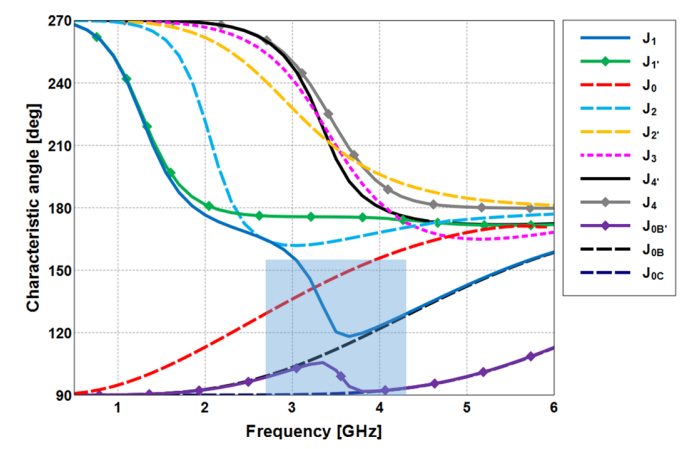

Fig. 6. Characteristic angle versus frequency for the first eleven modes of square metallic plate of length $L=80 \mathrm{~mm}$, with a slot of dimensions $L_{S}=33 \mathrm{~mm}$ and $W=1 \mathrm{~mm}$. The phenomenon of crossing avoidance is marked with a blue square.

The next step is to study the effect of inserting the slot at the middle of the square metallic plate already analyzed. The characteristic angle variation with frequency for the first eleven modes of this combined planar structure is presented in Fig. 6. If the modes obtained in Fig. 5 (isolated square metallic plate) are now compared with the modes of Fig. 6 , it is observed that pairs $J_{1}-J_{1}^{\prime}$, as well as $J_{4}-J_{4}$, are not degenerated any longer. This happens because the presence of the rectangular slot on the ground plane creates a meandering effect on the vertical current modes, and breaks the symmetry of the structure. Moreover, no new modes or extra resonances appear in the presence of the slot (Fig. 6), with respect to the case of the isolated square plate (Fig. 5). This means that the resonances of the magnetic modes of the slot shown in Section II, are not reflected by the electric modal analysis. Then, the electric modal analysis reveals that for the case of slotted planar antennas, the modes of the slot do not appear as independent current modes, but they merge with the electric current modes of the ground plane. Therefore, information about the slot resonances is somehow masked when an electric modal analysis is performed.

However, it has been observed that in the electric modal analysis of the slotted planar structure (Fig. 6), the phenomenon of crossing avoidance [15] occurs, exactly at the first resonance frequency of the slot (see shaded area in Fig. 6). For this particular case, the crossing avoidance exists due to the perturbation produced by the slot over the current distribution of the plate. This effect has been confirmed by an extensive analysis of slotted structures with different dimensions and shapes. Consequently, the phenomenon of crossing avoidance observed when slotted planar structures are analyzed with the electric modal approach, can be associated to the magnetic resonances of the slot.

\section{Slotted Planar Structure With FEEDING}

This section introduces the analysis when a voltage gap generator is placed in the center of the slot. In such case, this voltage gap excites the magnetic slot modes, which in turn excite the electric modes of the finite metallic ground plane. This effect, depending on the plate size, can reinforce the radiation and increase the impedance bandwidth as will be shown next.

As explained before, the voltage gap is inserted in the middle of the slot, exciting the magnetic modes associated to the slot. These magnetic modes of the slot couple to the finite ground plane creating electric current modes on the metallic surface. Fig. 7 shows the first magnetic current modes of the slot $\left(M_{n}\right)$ and their projection on electric current modes of the ground plane $\left(J_{n}\right)$. Due to the position of the voltage gap, only the magnetic modes of the slot with maximum magnetic current and even distribution will be excited. These even magnetic modes project on electric modes with zero electric current amplitude at the center of the slot.

Fig. 8 presents the simulated imaginary part of the input impedance of the slotted antenna showed in Fig. 4. As observed, the frequencies associated to the anti-resonances [16] correspond to the resonant frequencies of the magnetic modes $M_{1}$ and $M_{3}$, obtained in Section II for the slot on infinite ground plane. This behavior of the input reactance does not follow the same scheme as in the case of electrical structures, where resonances of the electric characteristic modes are associated to resonances of the input impedance. 


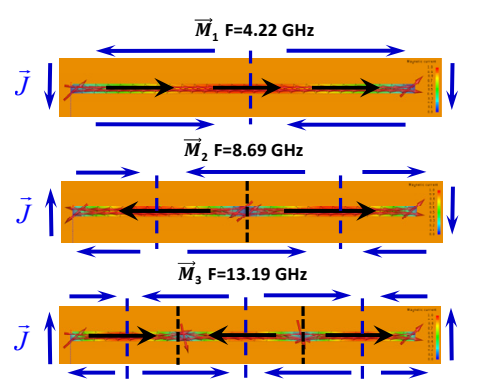

Fig. 7. Distribution of the magnetic characteristic currents marked in black and in blue its electrical one associated for the first three modes of a slot of $L_{S}=33 \mathrm{~mm}$ and $W=1 \mathrm{~mm}$, at their resonant frequency.

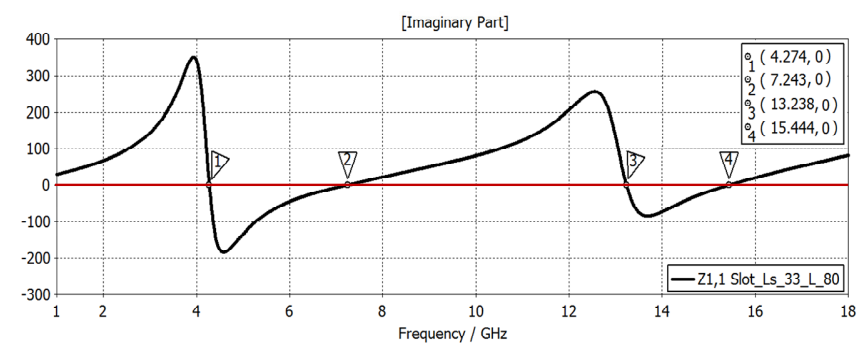

Fig. 8. Simulated imaginary part of the input impedance of the slotted planar antenna: $L_{S}=33 \mathrm{~mm}, W=1 \mathrm{~mm}$, and plate of $80 \times 80 \mathrm{~mm}^{2}, 0.35 \mathrm{~mm}$ thickness.

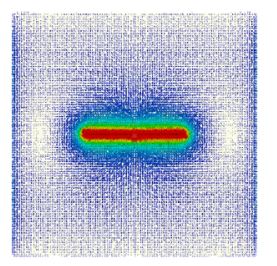

$\mathbf{f}=\mathbf{2} \mathbf{G H z}$

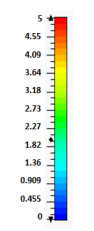

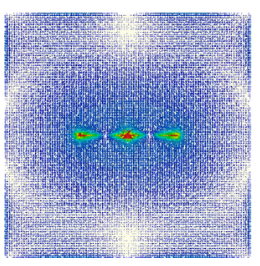

$\mathrm{f}=4.274 \mathrm{GHz}(1)$

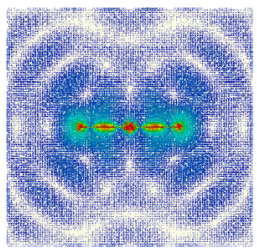

$\mathbf{f}=13.238 \mathrm{GHz}(3)$

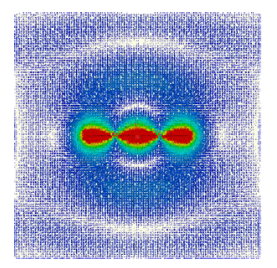

$\mathbf{f}=7.243 \mathrm{GHz}(2)$

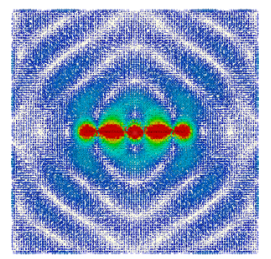

$f=15.445 \mathrm{GHz}(4)$
Fig. 9. Simulated total current distribution on the slotted planar antenna for the resonance and anti-resonance frequencies: $L_{S}=33 \mathrm{~mm}, W=1 \mathrm{~mm}$, and plate of $80 \times 80 \mathrm{~mm}^{2}, 0.35 \mathrm{~mm}$ thickness.

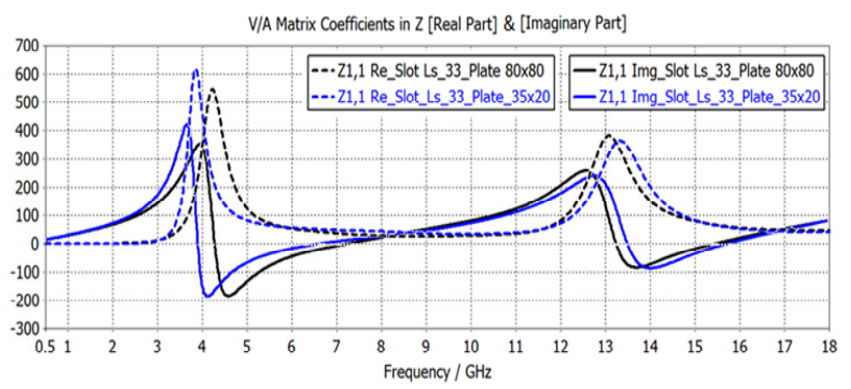

Fig. 10. Simulated imaginary and real part of the input impedance of the slotted planar antenna composed of a slot $\left(W=1 \mathrm{~mm}\right.$ and $\left.L_{S}=33 \mathrm{~mm}\right)$ in a square plate of: $80 \times 80 \mathrm{~mm}^{2}, 35 \times 20 \mathrm{~mm}^{2}$, and $0.35 \mathrm{~mm}$ thickness.

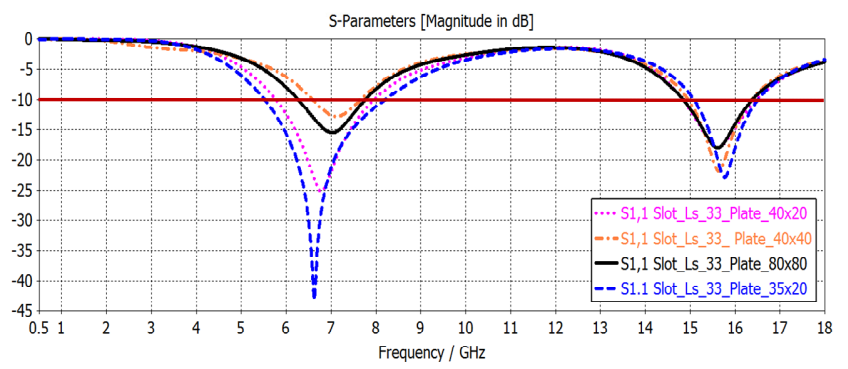

Fig. 11. Simulated reflection coefficient $(\mathrm{dB})$ of the slotted planar antenna composed of a slot ( $W=1 \mathrm{~mm}$ and $L_{S}=33 \mathrm{~mm}$ ) in a square plate of: $80 \times 80$ $\mathrm{mm}^{2}, 40 \times 40 \mathrm{~mm}^{2}, 40 \times 20 \mathrm{~mm}^{2}, 35 \times 20 \mathrm{~mm}^{2}$, and $0.35 \mathrm{~mm}$ thickness.

Moreover, it should be pointed out that these antiresonances fix the upper frequency up to which the magnetic modes of the slot will be excited. This is shown in Fig. 9, where the total current distribution in the slotted antenna is depicted. As observed, before the first anti-resonance at $\mathrm{f}=4.27 \mathrm{GHz}$, the total current is similar to that of mode $M_{l}$, so this mode is the responsible for radiation at the lowest frequencies. After the antiresonance, the total current resembles the distribution of mode $M_{3}$, and at first resonance ( $\mathrm{f}=7.24 \mathrm{GHz}$ ) mode $M_{3}$ is certainly the dominant mode in the total current distribution. The same occurs in the second antiresonance, in which the radiation of the slot changes from mode $M_{3}$ to $M_{5}$ immediately after the antiresonance. Therefore, resonances of the magnetic $\mathrm{CM}$ of the slot are responsible of the anti-resonances in the imaginary part of the input impedance of the slotted antenna. Then, in this case the resonant frequencies of the input impedance depend on the amplitude and distance between two consecutive antiresonances. The optimum dimensions for the plate when the slot is excited in the middle with a discrete port have been found to be: close to $\lambda_{s} / 2$ for the horizontal dimension, and $\lambda_{s} / 3$ for the vertical one. For the slot under study corresponds to $35 \mathrm{~mm}$ and $20 \mathrm{~mm}$ respectively.

Fig. 10 presents a comparison of the input impedance obtained for two plates of dimension $80 \times 80 \mathrm{~mm}^{2}$ and $35 \times 20$ $\mathrm{mm}^{2}$, where the last case corresponds to the optimum case proposed in this study. Note that the amplitude of the first anti-resonance has increased in the $35 \times 20 \mathrm{~mm}^{2}$ plate, producing a greater stability of the real part of the impedance around $50 \mathrm{Ohms}$ between both anti-resonances. This fact results in an increased radiation bandwidth and better matching, as shown in Fig. 11. With the proposed dimensions, bandwidth radiation increases from $21 \%$ for the $80 \times 80 \mathrm{~mm}^{2}$ plate until the $40 \%$ for the $35 \times 20 \mathrm{~mm}^{2}$ plate. As seen, anti-resonances are slightly shifted to lower frequencies with the plate size reduction. Specifically, the first antiresonance of the proposed plate moves to couple to the first ground plane resonance, reinforcing the radiation of the slot.

With the reduction of the plate size, the number of plate modes involved in the radiation of the structure is reduced [14] and consequently the excitation of non-desired higherorder modes is minimized. This effect can be verified in the total simulated current distribution of the $35 \times 20 \mathrm{~mm}^{2}$ plate showed in Fig. 12, when compared with the large plate shown previously in Fig. 9. In the comparison of the 
radiation patterns presented in Fig. 13, it is observed that the non-desired higher-order modes are responsible of the degradation of the radiation pattern of the $80 \times 80 \mathrm{~mm}^{2}$ slotted antenna, this effect is associated to the diffraction on the ground plane borders, and increases with frequency.

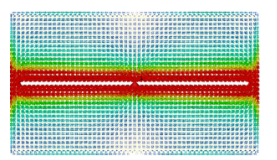

$\mathbf{f}=\mathbf{2} \mathbf{G H z}$

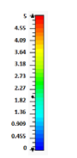

$\mathbf{f}=3.89 \mathrm{GHz}$

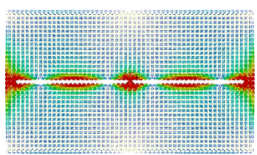

$\mathbf{f}=13.48 \mathrm{GHz}$

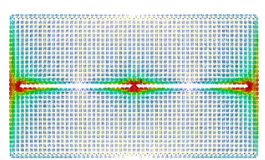

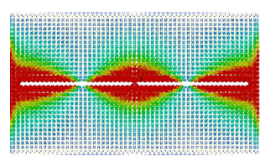

$f=6.61 \mathrm{GHz}$

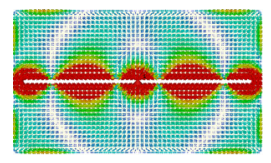

$\mathrm{f}=15.69 \mathrm{GHz}$
Fig. 12. Simulated total current distribution on the slotted planar antenna for the resonance and anti-resonance frequencies: $L_{S}=33 \mathrm{~mm}, W=1 \mathrm{~mm}$, and plate of $35 \times 20 \mathrm{~mm}^{2}$.
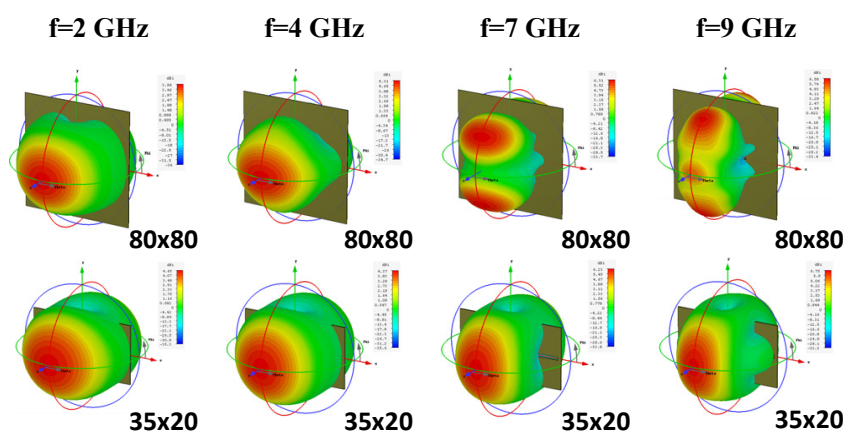

Fig. 13. Simulated 3D directivity radiation patterns of a slot $(W=1 \mathrm{~mm}$ and $L_{S}=33 \mathrm{~mm}$ ) in a square plate of: $80 \times 80 \mathrm{~mm}^{2}, 35 \times 20 \mathrm{~mm}^{2}$, and 0.35 mm thickness.

\section{CONCLUSIONS}

The electric CM analysis of slot antennas in finite ground plane reveals that the modes of the slot do not appears as independent modes, but they merge with the CM of the ground plane. However, the resonances of the slot can be identified in the CM analysis by the phenomenon of crossing avoidance. For the case of an infinite ground plane, the magnetic $\mathrm{CM}$ of the slot can be computed and identified. When the slot is excited with a voltage gap source, the resonances of these magnetic modes of the slot are reflected as anti-resonances of the input impedance of the slotted antenna. These anti-resonances fix the upper frequency of excited magnetic modes of the slotted antenna, while the resonances of the input impedance depend on the amplitude and distance between two consecutive anti-resonances. Therefore, after this study, it can be concluded that slots do not follow the same resonating behavior with $\mathrm{CM}$ as in electric structures like dipoles. Moreover, the finite plate size has a significant relevance in both the radiation behavior and the stability of the radiation pattern, and in the radiation bandwidth. Future research will focus on obtaining design guidelines for slotted planar antennas excited at different positions along the slot, with simple and multiple discrete feeds.

\section{ACKNOWLEDGMENT}

This work has been supported by the Spanish Ministry of Economy and Competitiveness (Ministerio de Economia y Competitividad) under the project TEC2016-78028-C3-3-P.

\section{REFERENCES}

[1] R. F. Harrington and J. R. Mautz, "Theory of Characteristic Modes for Conducting Bodies", IEEE Trans. Antennas and Propag., AP-19, 5, pp. 622-628, Sep. 1971.

[2] R. F. Harrington and J. R. Mautz, "Computation of characteristic modes for conducting bodies," IEEE Trans. Antennas Propagat., vol. AP-19, no. 5, pp. 629-639, September. 1971.

[3] M. Cabedo-Fabres, E. Antonino-Daviu, A. Valero-Nogueira and M. Ferrando-Bataller, "The Theory of Characteristic Modes Revisited: A contribution to the design of antennas for modern applications", IEEE Antennas and Propag. Mag., vol. 49, No. 5, pp.: 52 - 68. Oct. 2007.

[4] J. J. Adams, and J. T. Bernhard, "A Modal Approach to Tuning and Bandwidth Enhancement of an Electrically Small Antenna," IEEE Trans. on Antennas and Propagat., vol. 59, no. 4, pp. 1085-1092, 2011.

[5] Y. Chen and C. Wang, "Characteristic-mode-based improvement of circular polarized U-slot and E-shaped patch antennas," IEEE Antennas and Wireless Propagat. Lett., vol. 11, pp. 1474-1477, 2012.

[6] M. Sonkki, M. Cabedo-Fabrés, E. Antonino-Daviu, M. FerrandoBataller, and E.T. Salonen, "Creation of a Magnetic Boundary Condition in a Radiating Ground Plane to Excite Antenna Modes," IEEE Trans. Antennas Propagat., vol. 59, no. 10, pp. 3579- 3587, Oct. 2011.

[7] J. H. Lu and B. J. Huang, "Planar compact slot antenna with multiband operation for IEEE $802.16 \mathrm{~m}$ application," IEEE Trans. Antennas Propag., vol. 61, no. 3, pp. 1411-1414, Mar. 2013.

[8] R. Azadegan and K. Sarabandi, "A novel approach for miniaturization of slot antennas,” IEEE Trans. Antennas Propag., vol. 51, no. 3, pp. 421-429, Mar. 2003.

[9] E. Antonino-Daviu, M. Cabedo-Fabrés, M. Ferrando-Bataller, and V. M. Rodrigo-Peñarrocha, "Modal Analysis and Design of Bandnotched UWB Planar Monopole Antennas" IEEE Trans. Antennas Propagat., vol. 58, no. 5, pp. 1457-1467, May 2010.

[10] C. J. Wang, M.H. Shih and L. T. Chen, "A Wideband Open-Slot Antenna With Dual-Band Circular Polarization," IEEE Antennas Wireless Propag. Lett., vol. 14, pp. 1306-1309, 2015.

[11] R.F. Harrington and J. R. Mautz "Characteristic Modes for Aperture Problems," IEEE Transactions on Microwave Theory and Techniques, vol. MTT-33, no.6, pp. 500-505, June 1985.

[12] A. El-Hajj, and K. Y. Kabalan, "Characteristic Modes of a Rectangular Aperture in a Perfectly Conducting Plane,” IEEE Trans. on Antennas and Propagat., vol. 42, no. 10, October 1994.

[13] H. G.Booker, "Slot aerials and their relation to complementary wire aerials (Babinet's principle)" Part IIIA: Radiolocation, Journal of the Institution of Electrical Engineers, vol 93, no. 4, pp. 620-626, 1946.

[14] N. Mohamed Mohamed-Hicho, E. Antonino-Daviu, M. CabedoFabrés, and M. Ferrando-Bataller, "Chracteristic Modes in slot Antennas Etched in a Finite Ground Plane", IEEE International Symposium on Antennas and Propagation (APS), July 2016.

[15] K. R. Schab, J. M. Outwater, Jr., M. W. Young, and J. T. Bernhard, "Eigenvalue crossing avoidances in characteristic modes," IEEE Trans. on Antennas and Propagation, vol. 64, no. 7, pp. 2617-2627, July 2016.

[16] A. D. Yaghjian and S. R. Best, "Impedance, bandwidth, and Q of antennas," IEEE Trans. Antennas Propag., vol. 53, no. 4, pp. 12981324, Apr. 2005. 